\title{
New Battery Model and State-of-Health Determination Through Subspace Parameter Estimation and State-Observer Techniques
}

\author{
C. R. Gould, C. M. Bingham, Member, IEEE, D. A. Stone, and P. Bentley
}

\begin{abstract}
This paper describes a novel adaptive battery model based on a remapped variant of the well-known Randles' leadacid model. Remapping of the model is shown to allow improved modeling capabilities and accurate estimates of dynamic circuit parameters when used with subspace parameter-estimation techniques. The performance of the proposed methodology is demonstrated by application to batteries for an all-electric personal rapid transit vehicle from the Urban Light TRAnsport (ULTRA) program, which is designated for use at Heathrow Airport, U.K. The advantages of the proposed model over the Randles' circuit are demonstrated by comparisons with alternative observer/estimator techniques, such as the basic Utkin observer and the Kalman estimator. These techniques correctly identify and converge on voltages associated with the battery state-of-charge (SoC), despite erroneous initial conditions, thereby overcoming problems attributed to SoC drift (incurred by Coulomb-counting methods due to overcharging or ambient temperature fluctuations). Observation of these voltages, as well as online monitoring of the degradation of the estimated dynamic model parameters, allows battery aging (state-of-health) to also be assessed and, thereby, cell failure to be predicted. Due to the adaptive nature of the proposed algorithms, the techniques are suitable for applications over a wide range of operating environments, including large ambient temperature variations. Moreover, alternative battery topologies may also be accommodated by the automatic adjustment of the underlying state-space models used in both the parameter-estimation and observer/estimator stages.
\end{abstract}

Index Terms-Battery-management systems, energy storage, parameter estimation, system identification.

\section{INTRODUCTION}

$\mathbf{U}$ RBAN Light TRAnsport (ULTRA; see Fig. 1) is a personal rapid transit (PRT) vehicle developed by Advanced Transport Systems Ltd. [1]. It is an unmanned steer-by-wire electric vehicle capable of carrying a 500-kg payload at speeds up to $40 \mathrm{~km} / \mathrm{h}$. ULTRA aims to improve the transportation needs of a busy city or other public space (e.g., Heathrow Airport Terminal 5 in this case) with minimal environmental impact while providing a comfortable flexible travel solution for up to four passengers with luggage.

With the increasing drive toward use of X-by-wire control systems and the desire for passenger comfort (i.e., in-vehicle

Manuscript received November 1, 2008; revised May 1, 2009. Current version published October 2, 2009. This work was supported by the Engineering and Physical Sciences Research Council. The review of this paper was coordinated by Prof. A. Miraoui.

The authors are with the Electrical Machines and Drives Group, Department of Electronic and Electrical Engineering, University of Sheffield, Sheffield S10 2TN, U.K. (e-mail: c.gould@ shef.ac.uk).

Digital Object Identifier 10.1109/TVT.2009.2028348

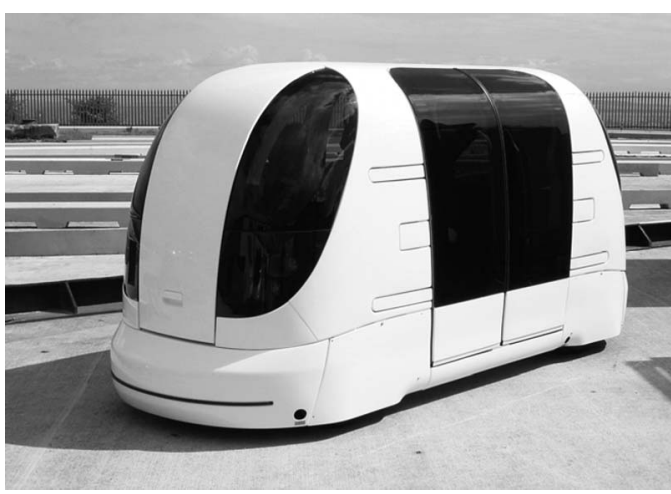

Fig. 1. ULTRA PRT vehicle [1].

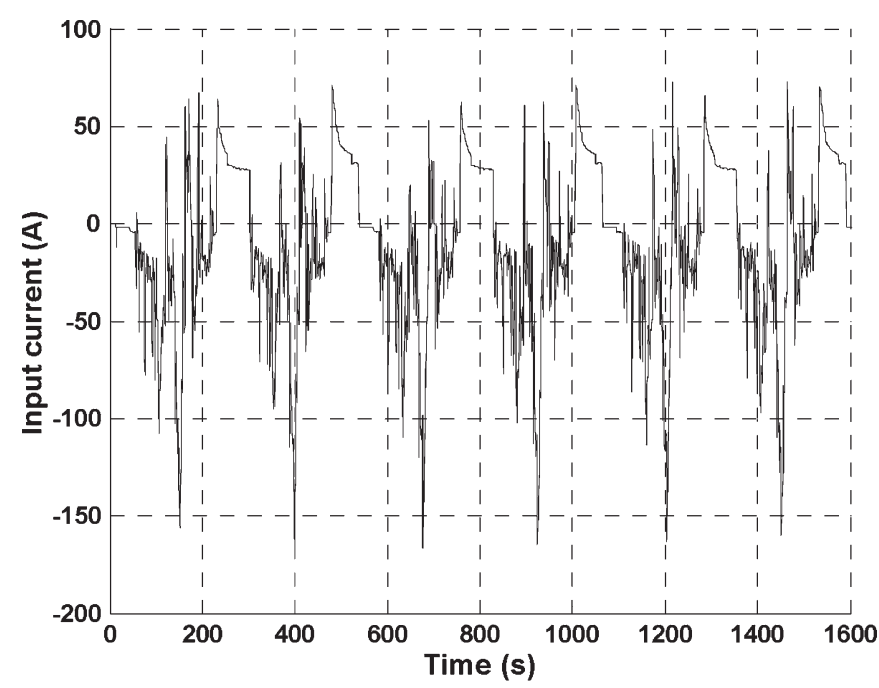

Fig. 2. Example of an ULTRA driving cycle current profile.

entertainment systems/air-conditioning), the large transient power demands (see Fig. 2) that are characteristic of such vehicles are becoming of utmost importance to the operational safety and reliability of the primary battery source, thereby necessitating the use of advanced battery state-of-function $(\mathrm{SoF})$ prediction techniques. Considering the many dynamic mechanisms occurring inside a battery to enable charge to be accepted and supplied [2], it is now necessary to assess the state-of-charge (SoC-amount of charge available in the battery at any time to sink/source excessive transients) and state-ofhealth (SoH-ability of that battery to repeatedly provide its rated capacity over time) of the battery in real time so that SoF 


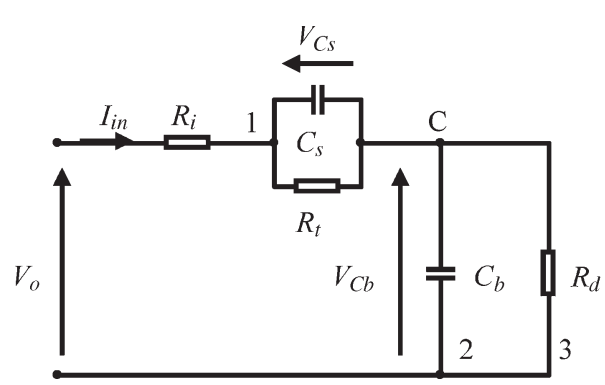

Fig. 3. Randles' lead-acid battery model.

can be assessed to avoid inconvenience to passengers and/or catastrophic failure of the safety-critical battery pack.

Whereas various SoC characterization techniques have been reported for lead-acid battery technologies [3]-[7], techniques for SoH determination are much less prevalent, primarily because $\mathrm{SoH}$ is a qualitative rather than a quantitative measure of battery aging or degradation [8], [9]. In most cases, SoH is estimated by monitoring the degradation of specific battery model parameters, as suggested in [7], where SoH deterioration is demonstrated using the well-known Randles' battery circuit model [10] using static parameters and a closed-loop extended Kalman filter [11]. However, for highly dynamic operational duties (e.g., Fig. 2), static parameter values are not sufficient to obtain accurate descriptions of battery behavior.

This paper therefore initially focuses on deriving an accurate dynamic model of battery systems when operating under high stress, large bipolar current transients (and for occasional opportunist-charging scenarios). It is shown that the Randles' model can be mapped to an alternative equivalent circuit, allowing subspace parameter-estimation algorithms to be applied online and in combination with both Utkin observer and Kalman estimator (KE) predictor/corrector techniques. The proposed model is demonstrated to be more appropriate than the Randles' circuit for use with the estimation algorithms, allowing adaptive identification and convergence of the model voltages associated with SoC and SoH monitoring. The resulting dynamic parameter estimates will also be shown to be useful for fault prediction and battery end-of-life determination.

\section{BATTERy MODELing}

The ULTRA vehicle relies on $4 \times 12-\mathrm{V}$ valve-regulated lead-acid (VRLA) batteries, nominally rated at 45-50 Ah. Each battery is individually monitored for SoF. Fig. 3 shows the Randles' circuit description of a cell, where $R_{d}$ represents the self-discharge resistance (approximately $5 \mathrm{k} \Omega$ ), and $C_{b}$ is considered the main charge store (equivalent to $\sim 1 \times 10^{5} \mathrm{~F}$ for a fully charged healthy $12-\mathrm{V}$ battery). The voltage across $C_{b}$ is considered to be a suitable indicator of SoC, whereas $\mathrm{SoH}$ is inferred by observing a significant change in $C_{b}$ over time due to aging effects such as active mass degradation [9] and crystallization of the active mass, which will effectively reduce the surface area of the parallel plates/grids. $R_{i}$ models the resistance of the battery's terminals and intercell connections (between 5-100 $\mathrm{m} \Omega$ ), whereas $R_{t}(10-500 \mathrm{~m} \Omega$ ) and $C_{s}(1000-20000 \mathrm{~F})$ describe transient effects resulting from shifting ion concentrations and plate current densities.

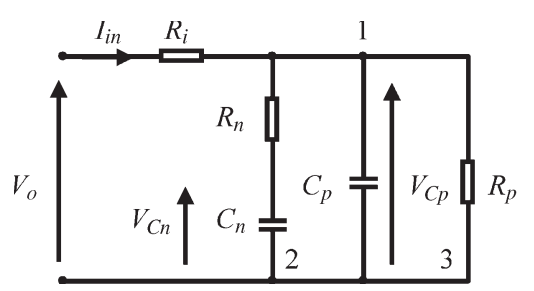

Fig. 4. Proposed remapped equivalent circuit.

Using a star-delta transform, the Randles' model can be mapped to the equivalent circuit shown in Fig. 4, where the star points of Fig. 3 are labeled 1, 2, and 3 with center C. Note that points 2 and 3 are shorted, and therefore, the resulting delta points will also require a short in parallel with the impedance usually expected.

The remaining two delta impedances are modeled by a capacitor and a resistor in series $\left(C_{n}\right.$ and $\left.R_{n}\right)$ and an impedance consisting of a capacitor and a resistor in parallel $\left(C_{p}\right.$ and $\left.R_{p}\right)$. The component and SoC voltage mappings are given in (1) and (2), respectively, and state equations are given in (3) and (4), respectively, for the Randles' and mapped circuits, i.e.,

$$
\begin{aligned}
C_{n} & =C_{b}^{2} /\left(C_{b}+C_{s}\right), \quad C_{p}=C_{b} C_{s} /\left(C_{b}+C_{s}\right) \\
R_{n} & =R_{t}\left(C_{b}+C_{s}\right)^{2} / C_{b}^{2}, \quad R_{p}=R_{d}+R_{t} \\
V_{C_{b}} & =\left(V_{C_{n}} C_{n}+V_{C_{p}} C_{p}\right) /\left(C_{n}+C_{p}\right) \\
\dot{V}_{C_{b}} & =\left(I_{\text {in }} R_{d}-V_{C_{b}}\right) / C_{b} R_{d} \\
\dot{V}_{C_{s}} & =\left(I_{\text {in }} R_{t}-V_{C_{s}}\right) / C_{s} R_{t} \\
V_{o} & =V_{C_{s}}+V_{C_{b}}+I_{\text {in }} R_{i} \\
\dot{V}_{C_{p}} & =\frac{V_{C_{n}}}{R_{n} C_{p}}-\frac{V_{C_{p}}\left(R_{n}+R_{p}\right)}{C_{p} R_{n} R_{p}}+\frac{I_{\text {in }}}{C_{p}} \\
\dot{V}_{C_{n}} & =-\frac{V_{C_{n}}}{R_{n} C_{n}}+\frac{V_{C_{p}}}{C_{n} R_{n}}, \quad V_{o}=V_{C_{p}}+I_{\text {in }} R_{i} .
\end{aligned}
$$

The validity of the mapping and advantages of the technique will be demonstrated in the next section using subspace parameter estimation, initially on a system of known parameters for model comparison, and finally on experimental data captured from tests on a battery.

\section{Subspace Parameter Estimation}

\section{A. Theory}

The input-output and state relationships of a discrete-time system modeled using $A_{d}, B_{d}, C_{d}$, and $D_{d}$ and white noise inputs $w(t)$ and $v(t)(5)$ can be transformed using any invertible matrix $T$ (6). It can be shown (Ljung 10.80-10.129 [12]) that parameters of the system matrices $A_{d}, B_{d}, C_{d}$, and $D_{d}$ and initial conditions $\widetilde{x}(0)$ can be estimated by applying subspace parameter-estimation techniques if sufficient (and appropriate) data are available.

For brevity, the process of solving the subspace algorithms and choosing the correct weighting factors for a leastsquares solution is omitted here; see the extensive treatment given by Ljung [12] for details. In essence, the subspace 
parameter-estimation algorithm allows static battery model parameters to be estimated for a "window" of input-output data $\left(I_{\text {in }}\right.$ and $V_{o}$ ). By using a "rolling" window as data are captured, an approximation of the dynamic battery model parameters can be obtained that accommodates transient electrochemical and thermal effects. In practice, this is achieved by storing the collected input-output data in a first-in-first-out (FIFO) buffer of suitable size and is updated at each sample step. Parameter estimation is then performed on the contents of the buffer at a lower rate than that of data sampling to decrease computational complexity. Due to the large time constants involved with typical combinations of $C_{b}$ and $R_{d}$ (or $C_{n}$ and $R_{p}$ ), it is not practical to acquire sufficient buffered data for accurate estimation of the exact values of these large components without neglecting other more transient parameter variations (i.e., occasional charging periods), and thus, a structured subspace estimation model is used in which the time constant associated with the product $C_{b} R_{d}$ (or $C_{n} R_{n}$ ) is assumed to be large (compared with the time constant associated with $C_{s} R_{t}$ or $C_{p} R_{n}$ ) and, therefore, subject to a negligible change over a small buffer of data (100 $\mathrm{s}$ in this case). Likewise, $R_{d}$ is assumed to be sufficiently large $(5 \mathrm{k} \Omega)$ so that a negligible current is drawn by the discharge resistance, thereby also leading to the assumption that $\left(R_{n}+R_{p}\right) / R_{p} \rightarrow 1$ in (4).

This results in the modified state-space models for the Randles' and remapped circuits shown in (7) and (8), respectively. The aforementioned equations are given as follows:

$$
\begin{aligned}
x(t+1) & =A_{d} x(t)+B_{d} u(t)+w(t) \\
y(t) & =C_{d} x(t)+D_{d} u(t)+v(t) \\
\widetilde{x}(t+1) & =T^{-1} A_{d} T \widetilde{x}(t)+T^{-1} B_{d} u(t)+\widetilde{w}(t) \\
y(t) & =C_{d} T \widetilde{x}(t)+D_{d} u(t)+v(t) \\
\dot{V}_{C_{b}} & =\frac{I_{\mathrm{in}}}{C_{b}}, \quad \dot{V}_{C_{s}}=-\frac{V_{C_{s}}}{C_{s} R_{t}}+\frac{I_{\mathrm{in}}}{C_{s}} \\
V_{o} & =V_{C_{s}}+V_{C_{b}}+I_{\mathrm{in}} R_{i} \\
\dot{V}_{C_{n}} & =-\frac{V_{C_{n}}}{R_{n} C_{n}}+\frac{V_{C_{p}}}{C_{n} R_{n}} \\
\dot{V}_{C_{p}} & =\frac{V_{C_{n}}}{R_{n} C_{p}}-\frac{V_{C_{p}}}{C_{p} R_{n}}+\frac{I_{\mathrm{in}}}{C_{p}} \\
V_{o} & =V_{C_{p}}+I_{\mathrm{in}} R_{i} .
\end{aligned}
$$

\section{B. Comparison of Simulated Subspace Estimation Using Randles' and Mapped Circuit Models}

To investigate the performance of subspace parameter estimation for each model, equivalent static parameters are initially assigned to each model [using (1)] so that a current demand (see Fig. 5) consisting of a series of the cycles shown in Fig. 2 can be applied to each battery model. Due to the one-to-one mapping, the battery terminal voltages of each model will be the same. The current demand and resulting terminal voltages are then applied to the subspace parameter-estimation scheme to estimate the parameters of each model.

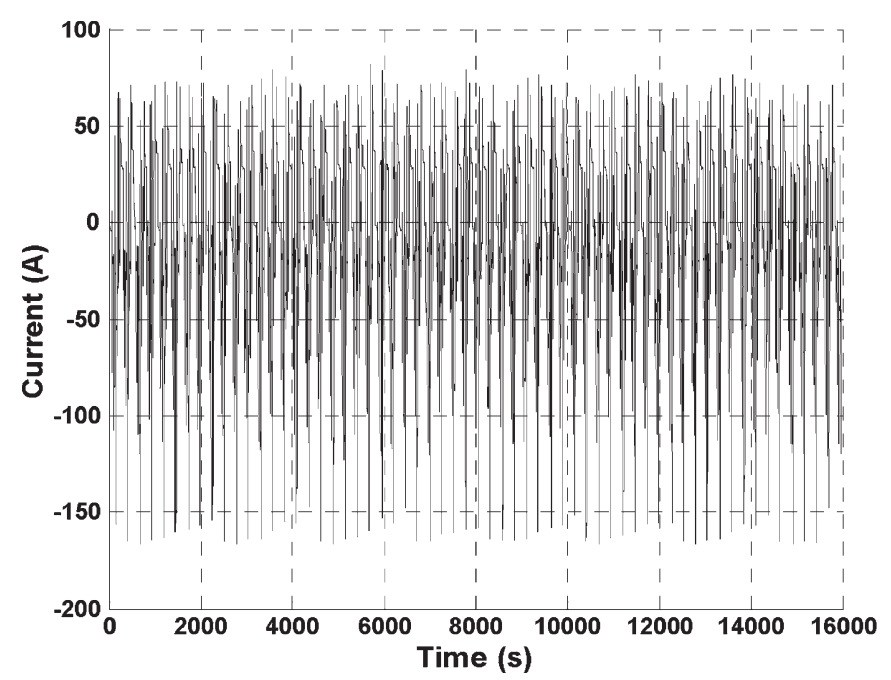

Fig. 5. Typical ULTRA current demand to fully discharge a single 48-Ah VRLA battery.

TABLE I

INITIAL MODEL PARAMETERS

\begin{tabular}{|c|c|c|c|}
\hline $\begin{array}{c}\text { Randles' } \\
\text { parameter }\end{array}$ & Randles'value & $\begin{array}{c}\text { Mapped } \\
\text { parameter }\end{array}$ & Mapped value \\
\hline$C_{b}$ & $90000 \mathrm{~F}$ & $C_{n}$ & $85263 \mathrm{~F}$ \\
\hline$C_{S}$ & $5000 \mathrm{~F}$ & $C_{p}$ & $4736 \mathrm{~F}$ \\
\hline$R_{i}$ & $80 \mathrm{~m} \Omega$ & $R_{i}$ & $80 \mathrm{~m} \Omega$ \\
\hline$R_{t}$ & $30 \mathrm{~m} \Omega$ & $R_{n}$ & $33 \mathrm{~m} \Omega$ \\
\hline$R_{d}$ & $5 \mathrm{k} \Omega$ & $R_{p}$ & $5 \mathrm{k} \Omega$ \\
\hline
\end{tabular}

Following the determination of initial model parameters (see Table I), the identification algorithms are subsequently applied using the data shown in Fig. 5, which gives a typical ULTRA current demand profile applied to a single 12-V 48-Ah VRLA battery at $100 \%$ SoC (approximately $13.2 \mathrm{~V}$ open-circuit (cct) terminal voltage), to take it to almost full discharge at an average rate of $C / 5$.

Parameter estimates from the data and the resulting terminal voltage from the models are obtained by sampling the data at $1 \mathrm{~Hz}$ and storing it in a two-channel 100-s FIFO buffer. To reduce computational complexity, the contents of the buffer undergo parameter estimation every $10 \mathrm{~s}$. Consequently, each time the parameter estimation algorithm is performed, the static parameters obtained from $100 \mathrm{~s}$ of data are applied to the battery model for a period of $10 \mathrm{~s}$ prior to the next parameterestimation cycle. This allows the model parameters associated with larger time constants of the systems to be assumed static over each successive 100-s period of data, since the capacitance associated with the main charge store will negligibly vary over this period.

Since it is difficult to predict the variation in model parameters over time, fixed initial parameters are used for each buffer (see Table II), whereas the initial conditions of the states (i.e., the voltages associated with each capacitor) can be estimated by integrating the current flowing into the main charge store and calculating the voltage across the (provisionally estimated) capacitance. Initial states/conditions used for the first buffer are taken from steady-state open-cct terminal voltage readings. 
TABLE II

FIXED INITIAL PARAMETERS

\begin{tabular}{|c|c|c|c|}
\hline $\begin{array}{c}\text { Randles' } \\
\text { parameter }\end{array}$ & $\begin{array}{c}\text { Randles' initial } \\
\text { condition }\end{array}$ & $\begin{array}{c}\text { Mapped } \\
\text { parameter }\end{array}$ & $\begin{array}{c}\text { Mapped initial } \\
\text { condition }\end{array}$ \\
\hline$C_{b}$ & $92000 \mathrm{~F}$ & $C_{n}$ & $90000 \mathrm{~F}$ \\
\hline$C_{s}$ & $2050 \mathrm{~F}$ & $C_{p}$ & $2000 \mathrm{~F}$ \\
\hline$R_{i}$ & $70 \mathrm{~m} \Omega$ & $R_{i}$ & $70 \mathrm{~m} \Omega$ \\
\hline$R_{t}$ & $29 \mathrm{~m} \Omega$ & $R_{n}$ & $30 \mathrm{~m} \Omega$ \\
\hline $\mathrm{VC}_{\mathrm{b}}(0)$ & $13.37 \mathrm{~V}$ & $\mathrm{VC}_{\mathrm{n}}(0)$ & $13.37 \mathrm{~V}$ \\
\hline $\mathrm{VC}_{\mathrm{s}}(0)$ & $0 \mathrm{~V}$ & $\mathrm{VC}_{\mathrm{p}}(0)$ & $13.37 \mathrm{~V}$ \\
\hline
\end{tabular}

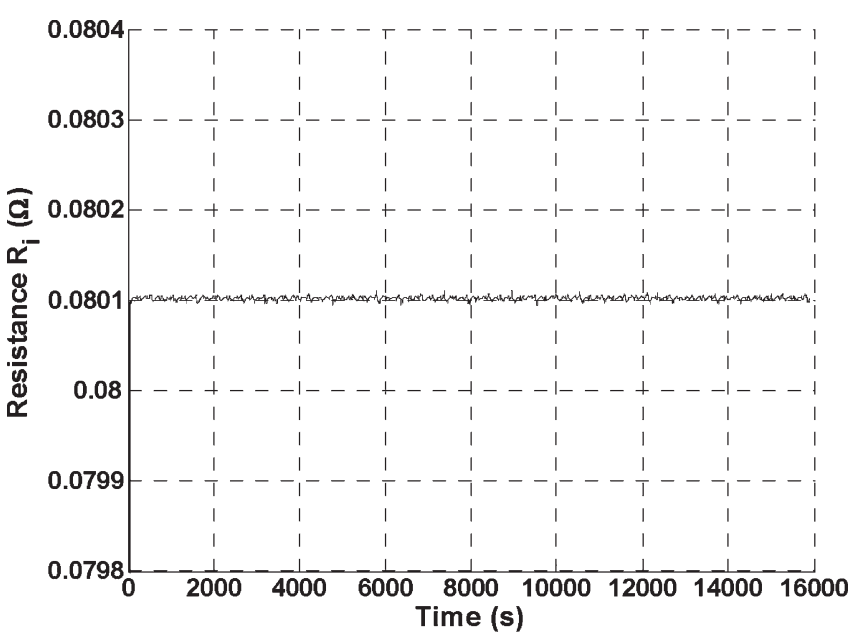

Fig. 6. Mapped model subspace parameter estimate of $\sim 80 \mathrm{~m} \Omega R_{i}$.

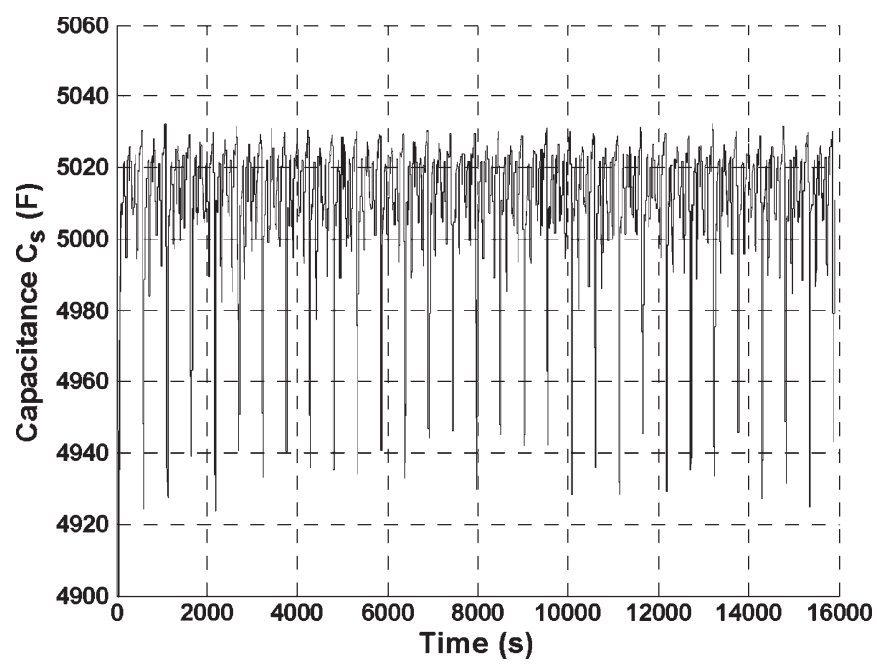

Fig. 7. Mapped model subspace parameter estimate of $\sim 5000 \mathrm{~F} C_{s}$.

Figs. 6-8 show the resulting estimates of the static parameters when using the remapped equivalent circuit model and (1) to postprocess the results for comparison with the parameters from Fig. 3 (i.e., the Randles' static parameters from Table I). Application of the parameter-estimation technique to both models can be seen to correctly identify the average static parameters for each buffer of data, given the initial conditions recorded in Table II.

For a direct comparison between the two modeling approaches and the predefined static parameters, Table III demonstrates the relative accuracy of each model, including the

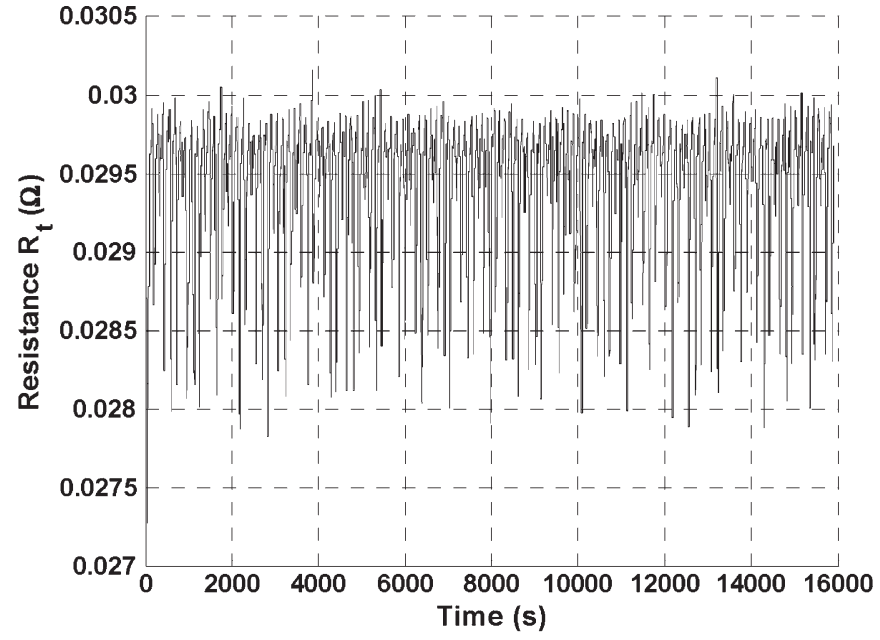

Fig. 8. Mapped model subspace parameter estimate of $\sim 30 \mathrm{~m} \Omega R_{t}$.

standard deviation of the percentage error in each parameter from the two models. Initially, it seems that the two models are equally as accurate, particularly for estimating the terminal resistance $R_{i}$. However, advantages can be seen when using the mapped circuit model for the estimation of the components associated with the electrochemical transient effects within the battery, due to the mapped model having two states in parallel as opposed to the series circuit combination of states seen in the Randles' model. This will further be highlighted when the parameter-estimation techniques are combined with predictor/corrector algorithms to monitor the models' circuit voltages associated with SoF.

The subspace parameter estimation algorithm is now applied to experimental data collected from application of the current demand of Fig. 5 (via a personal computer-based LabVIEW hardware development platform) to a fully charged well-conditioned 12-V 48-Ah (nominal) Monobloc battery, resulting in the measured terminal voltage waveform shown in Fig. 9. The battery under test is housed in an environmentally controlled test chamber (see Fig. 10), whereas the terminal voltage, current demand, and terminal temperature are monitored by a dSPACE system, which implements the online estimator algorithms.

In parallel with the estimator algorithms, dSPACE also calculates the cell-discharge characteristic to calculate the amperehours being drawn from the battery (see Fig. 11). Typically, this characteristic is normalized to the nominal total capacity of the battery to monitor SoC. However, it will be shown that this type of "Coulomb-counting" method proves inaccurate as the total capacity of the battery varies with SoH.

Examination of the experimental waveform in Fig. 9 clearly demonstrates the variation in battery parameters that must be occurring due to SoF of the battery. Of particular interest are portions of the waveform showing excessive transient activity near to full-charge and deep-discharge areas of operation. Intuitively, these periods mark situations where either an almost fully charged battery is subject to charging periods in excess of its maximum capacity, thereby provoking "gassing" reactions within the battery or, alternatively, an almost discharged battery being forced to source transient power that it no longer has the 
TABLE III

ACCuracy of the Randles' and the Proposed Model Relative to the FiXed Initial Parameters of Table III

\begin{tabular}{|c|c|c|c|c|c|c|}
\hline \multirow{3}{*}{$\begin{array}{l}\text { N.B. Relative to static } \\
\text { parameters in Table I }\end{array}$} & \multicolumn{6}{|c|}{ Model parameter } \\
\hline & \multicolumn{2}{|c|}{$R_{i}$} & \multicolumn{2}{|c|}{$C_{s}$} & \multicolumn{2}{|c|}{$R_{t}$} \\
\hline & Randles' & Mapped & Randles' & Mapped & Randles' & Mapped \\
\hline Average error $(\%)$ & 0.123 & 0.123 & 0.108 & 0.108 & 1.283 & 1.949 \\
\hline Maximum error $(\%)$ & 0.138 & 0.138 & 2.950 & 1.530 & 22.939 & 7.225 \\
\hline Standard deviation of error & $2.53 \mathrm{e}-3$ & $2.51 \mathrm{e}-3$ & 0.324 & 0.372 & 2.070 & 1.578 \\
\hline
\end{tabular}

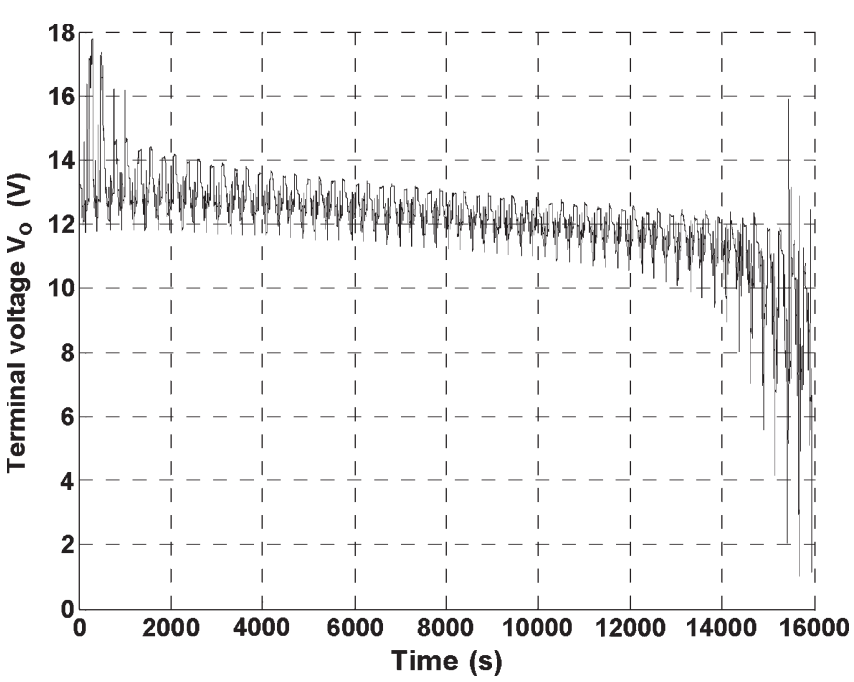

Fig. 9. Experimental terminal voltage waveform from 12-V 48-Ah (nominal) battery subjected to the current demand of Fig. 5 .

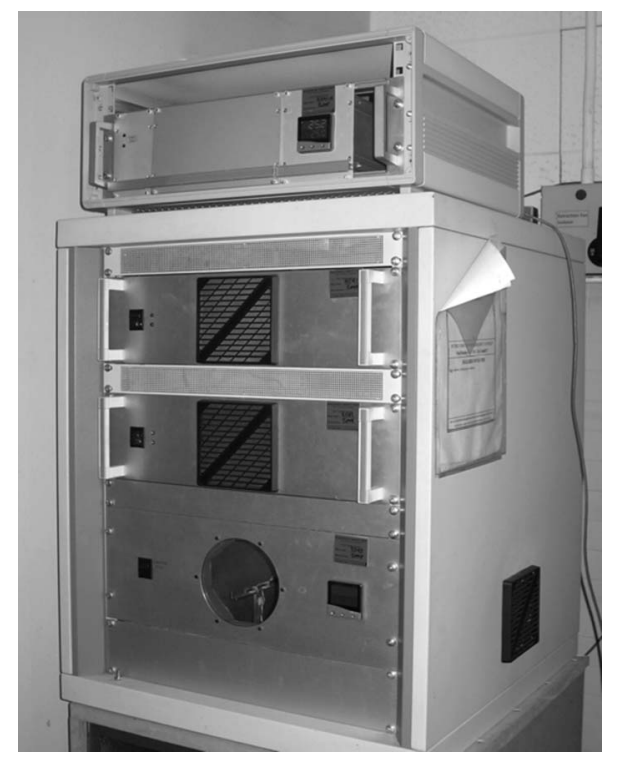

Fig. 10. Environmentally controlled test chamber.

capacity to provide. Both situations should be avoided to ensure good SoF and extended lifetime of a battery.

Usually, the battery is only operated between $80 \%$ and $40 \%$ SoC (i.e., Time $=2000 \mathrm{~s}-$ Time $=12000 \mathrm{~s}$ in Fig. 9) to ameliorate problems associated with these situations. However, from an efficiency perspective, it would be desirable to identify the widest operating region for each battery, and this can be achieved by monitoring the variation in parameters that

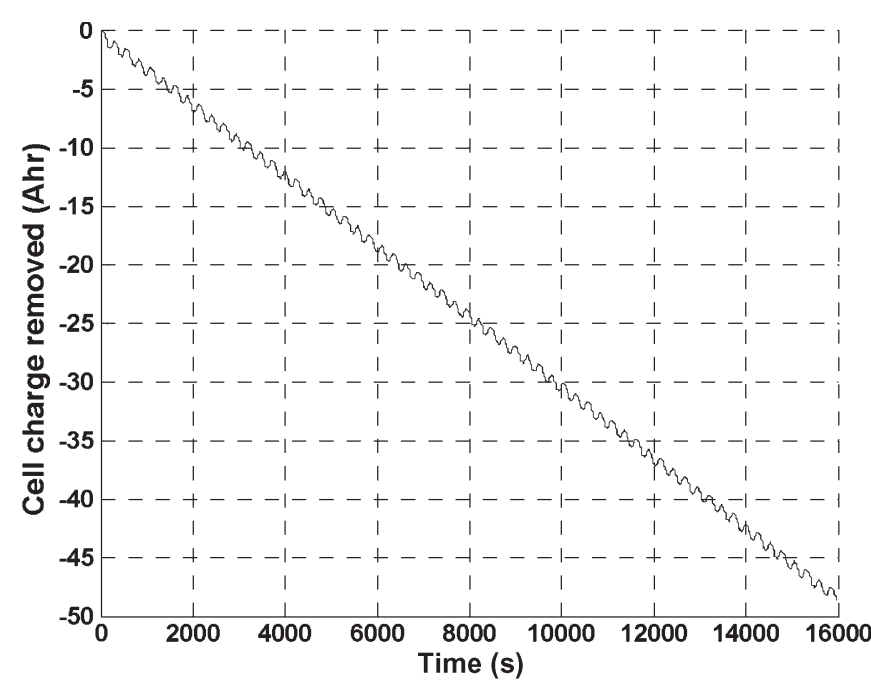

Fig. 11. Resulting cell discharge characteristic.

TABLE IV

SubsPaCe PARAMETER INITIAL CONDITIONS

\begin{tabular}{|c|c|c|c|}
\hline $\begin{array}{c}\text { Randles' } \\
\text { parameter }\end{array}$ & $\begin{array}{c}\text { Randles' initial } \\
\text { condition }\end{array}$ & $\begin{array}{c}\text { Mapped } \\
\text { parameter }\end{array}$ & $\begin{array}{c}\text { Mapped initial } \\
\text { condition }\end{array}$ \\
\hline$C_{b}$ & $90000 \mathrm{~F}$ & $C_{n}$ & $90000 \mathrm{~F}$ \\
\hline$C_{s}$ & $2000 \mathrm{~F}$ & $C_{p}$ & $2000 \mathrm{~F}$ \\
\hline$R_{i}$ & $10 \mathrm{~m} \Omega$ & $R_{i}$ & $10 \mathrm{~m} \Omega$ \\
\hline$R_{t}$ & $10 \mathrm{~m} \Omega$ & $R_{n}$ & $10 \mathrm{~m} \Omega$ \\
\hline$V C_{b}(0)$ & $13.37 \mathrm{~V}$ & $V C_{n}(0)$ & $13.37 \mathrm{~V}$ \\
\hline$V C_{s}(0)$ & $0 \mathrm{~V}$ & $V C_{p}(0)$ & $13.37 \mathrm{~V}$ \\
\hline
\end{tabular}

result from application of the proposed parameter-estimation technique.

\section{Experimental Results for Subspace Parameter Estimation Using Randles' and Remapped Models}

Application of the parameter estimation algorithm (with initial conditions given in Table IV) to the input-output data shown in Figs. 5 and 9 provides the estimates of battery parameters shown in Figs. 12-14.

From the results, areas of operation that may degrade battery performance (such as overcharge/excessive discharge situations) can readily be identified.

To determine variations in $\mathrm{SoC}$ and $\mathrm{SoH}$, it is necessary to estimate the voltage across the main charge store $C_{b}$. Although the use of dynamic parameter estimates allows more accurate "open-loop" estimation of this voltage than with the use of static parameters, it is evident from Figs. 12-14 that some of the parameter combinations or initial state conditions can 


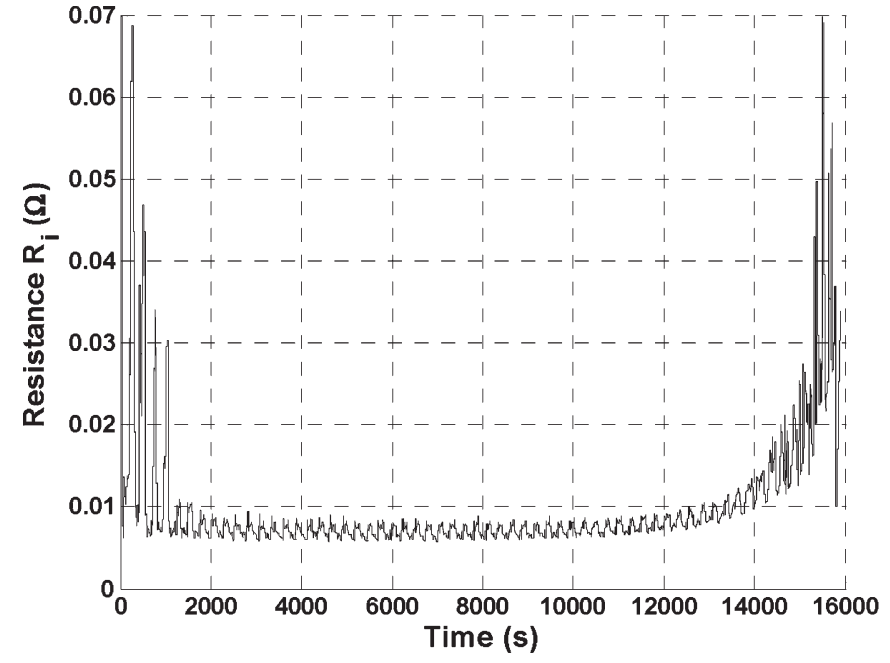

(a)

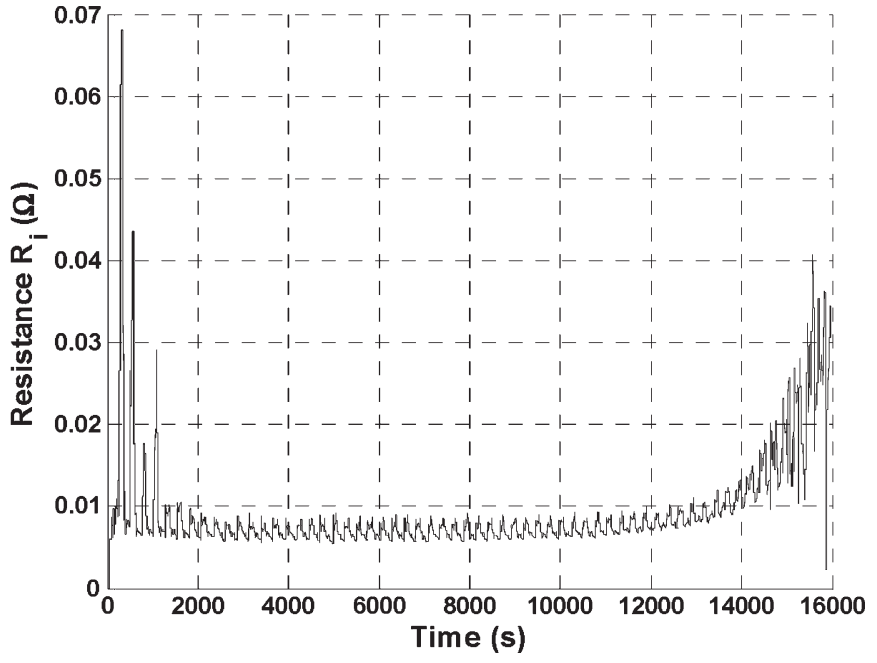

(b)

Fig. 12. Online battery parameter $R_{i}$ (a) from the Randles' model and (b) from the mapped circuit model.

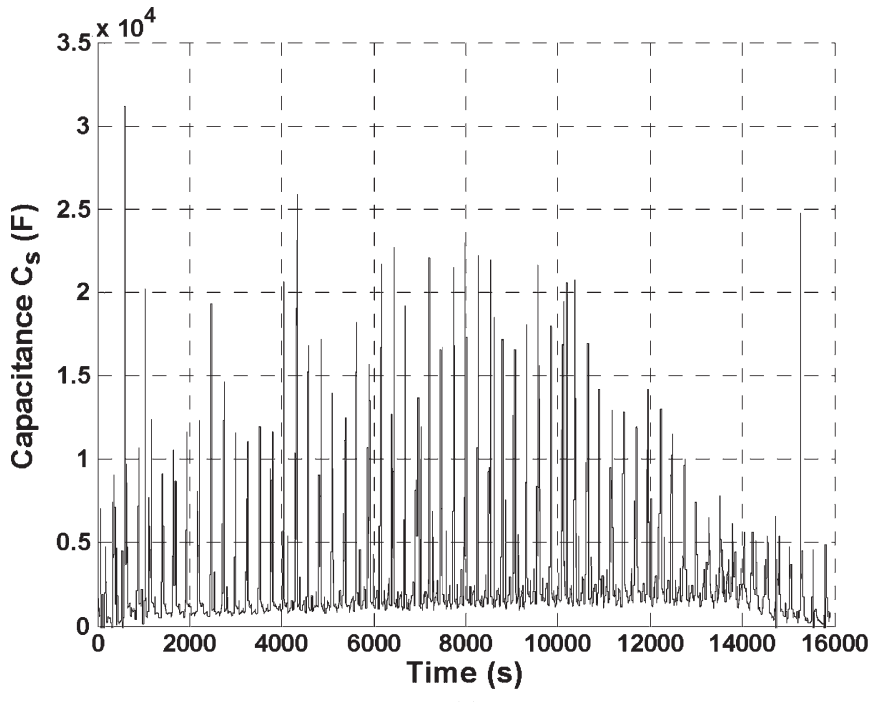

(a)

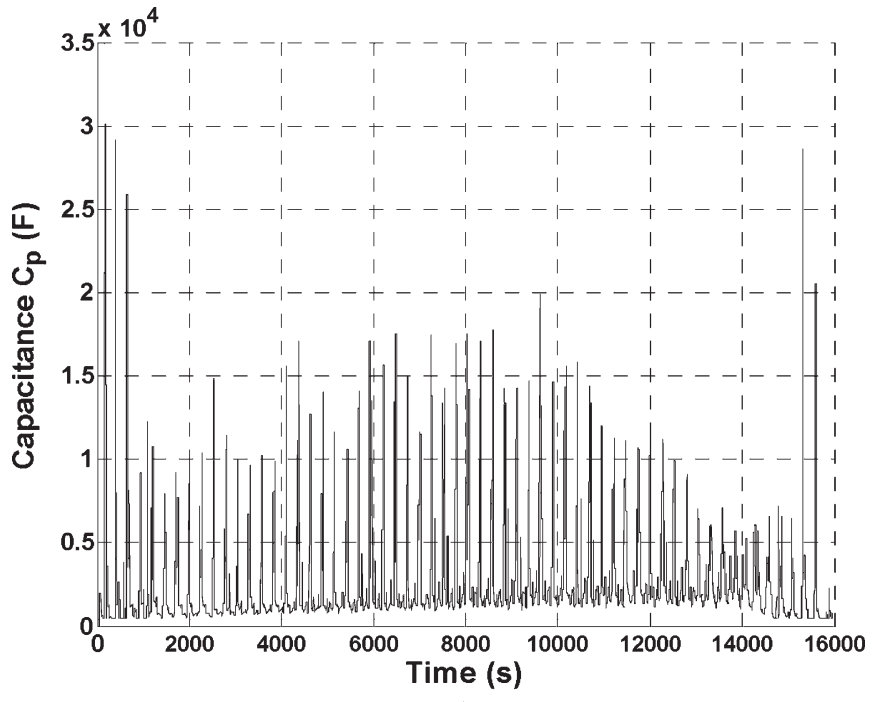

(b)

Fig. 13. Online battery parameter (a) $C_{s}$ from the Randles' model and (b) $C_{p}$ from the mapped circuit model.

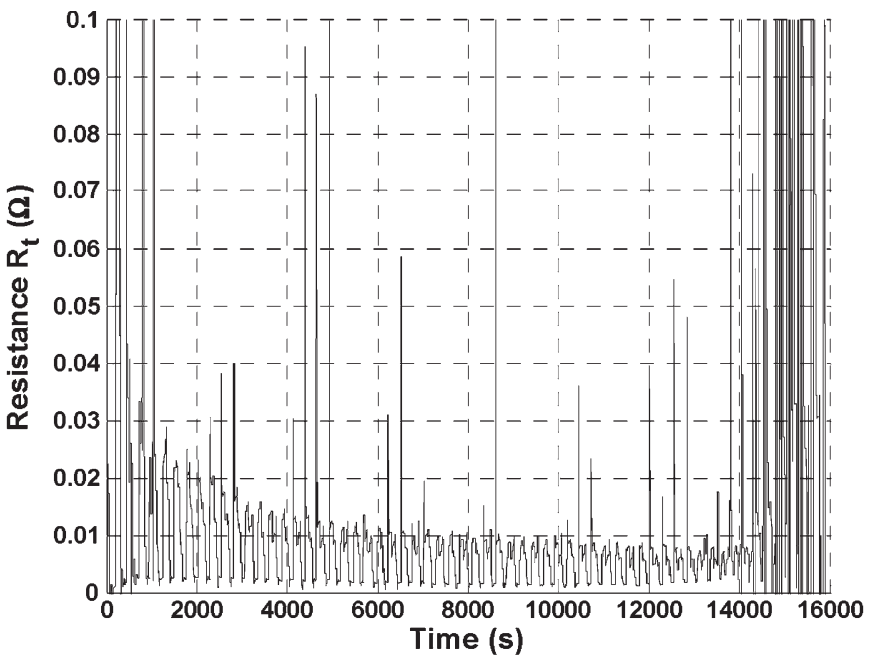

(a)

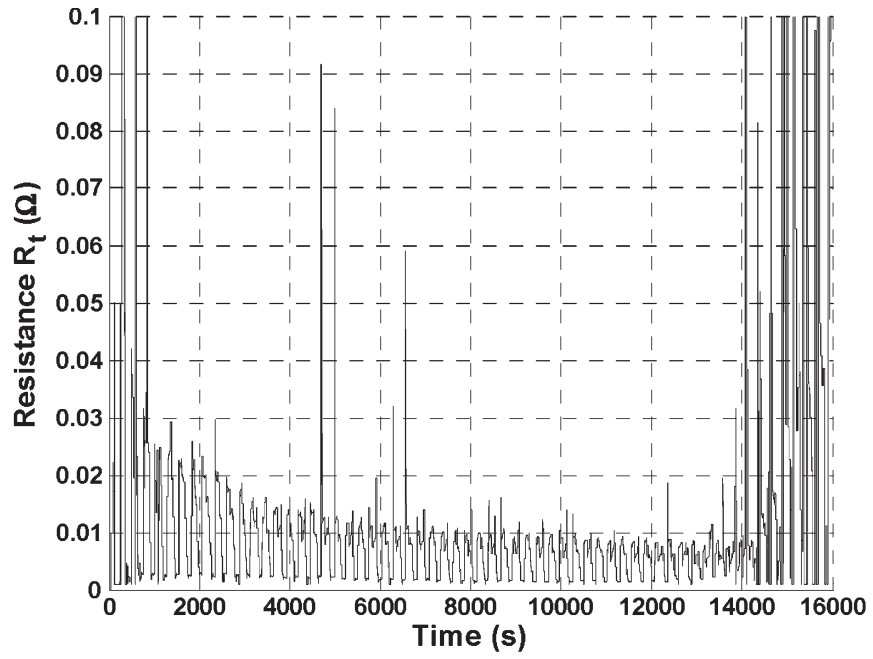

(b)

Fig. 14. Online battery parameter (a) $R_{t}$ from the Randles' model and (b) $R_{n}$ from the mapped circuit model. 
produce unuseful results, which could introduce SoC "drift" similar to that incurred by traditional current integration or Coulomb-counting techniques. To correctly identify and converge onto the correct circuit voltages, therefore, it is proposed to use a state-space observer technique [7], [11], where "best decisions" are recursively made to account for previous- and current-model parameters, associated errors, input-output data, and system noise. Candidate observers often make use of predictor/corrector-type mechanisms, whereby an initial solution to the problem is calculated using some or no previous information and then recursively corrected over time using weighted gain terms applied to different states until a "best solution" is reached.

Two examples of such observer/estimator techniques are now investigated for their suitability for this application.

\section{SLIDING-Mode OBSERVER THEORY}

\section{A. Underlying Theory}

An uncertain dynamical system description (9) may be "observed" using a discontinuous observer of the form of (10) proposed by Utkin in 1981, where $\hat{x}_{1}$ and $\hat{y}$ are estimates of $x_{1}$ and $y$, whereas $L \in \Re$ and $M \in+\Re$ are feedback gains. This results in the discontinuous observer form given in (11) for the Randles' model and in (12) for the remapped circuit model. Starting from a known initial condition (usually determined from the open-cct terminal voltage), at each sampling instant, (11) and (12) are applied to each model using parameters that are updated every $10 \mathrm{~s}$ (supplied from the subspace parameter estimation scheme). The aforementioned equations are given as follows:

$$
\begin{aligned}
& \dot{x}(t)=A x(t)+B u(t)+f(x, u, t), \quad y(t)=C x(t) \\
& \dot{\hat{x}}_{1}=A_{11} \hat{x}_{1}+A_{12} \hat{y}+B_{1} u+L v_{1} \\
& \dot{\hat{y}}=A_{21} \hat{x}_{1}+A_{22} \hat{y}+B_{2} u-v_{2} \\
& v_{i}=M \operatorname{sgn}(\hat{y}-y) \\
& {\left[\begin{array}{c}
\dot{V}_{C_{b}} \\
\dot{V}_{C_{s}} \\
\dot{V}_{\text {eq }}
\end{array}\right]=\left[\begin{array}{ccc}
-1 / C_{b} R_{d} & 0 & 0 \\
0 & -1 / C_{s} R_{t} & 0 \\
-1 / C_{b} R_{d} & -1 / C_{s} R_{t} & 0
\end{array}\right]\left[\begin{array}{c}
V_{C_{b}} \\
V_{C_{s}} \\
V_{\text {eq }}
\end{array}\right]} \\
& +\left[\begin{array}{c}
1 / C_{b} \\
1 / C_{s} \\
\left(C_{b}+C_{s}\right) / C_{b} C_{s}
\end{array}\right] I_{\text {in }} \\
& +\left[\begin{array}{c}
L_{1} M \operatorname{sgn}\left(\hat{y}-V_{\text {eq }}-I_{\text {in }} R_{i}\right) \\
L_{2} M \operatorname{sgn}\left(\hat{y}-V_{\text {eq }}-I_{\text {in }} R_{i}\right) \\
-M_{1} \operatorname{sgn}\left(\hat{y}-V_{\text {eq }}-I_{\text {in }} R_{i}\right)
\end{array}\right] \\
& V_{o}=V_{\mathrm{eq}}+I_{\mathrm{in}} R_{i} \\
& {\left[\begin{array}{c}
\dot{V}_{C_{n}} \\
\dot{V}_{C_{p}}
\end{array}\right]=\left[\begin{array}{cc}
-1 / R_{n} C_{n} & 1 / R_{n} C_{n} \\
1 / R_{n} C_{p} & -\left(R_{n}+R_{p}\right) / R_{n} C_{p} R_{p}
\end{array}\right]\left[\begin{array}{c}
V_{C_{n}} \\
V_{C_{p}}
\end{array}\right]} \\
& +\left[\begin{array}{c}
0 \\
1 / C_{p}
\end{array}\right] I_{\text {in }}+\left[\begin{array}{c}
L_{3} M \operatorname{sgn}\left(\hat{y}-V_{C_{p}}-I_{\text {in }} R_{i}\right) \\
-M_{2} \operatorname{sgn}\left(\hat{y}-V_{C_{p}}-I_{\text {in }} R_{i}\right)
\end{array}\right] \\
& V_{o}=V_{C_{p}}+I_{\mathrm{in}} R_{i} \text {. }
\end{aligned}
$$

\section{B. Results for Utkin Observer Estimation of SoC for Both Randles' and Remapped Models}

Both the parameter estimation and observer schemes are initialized with $L_{1}=-1, L_{2}=-0.002, L_{3}=-1, M_{1}=0.02$, $M_{2}=0.02, R_{i}=6 \mathrm{~m} \Omega, R_{t}=10 \mathrm{~m} \Omega, R_{d}=5 \mathrm{k} \Omega, C_{s}=$ $2000 \mathrm{~F}, C_{b}=100000 \mathrm{~F}, V_{C_{s}}(0)=0 \mathrm{~V}, R_{n}=10 \mathrm{~m} \Omega, R_{p}=$ $5 \mathrm{k} \Omega, C_{p}=2000 \mathrm{~F}, C_{n}=100000 \mathrm{~F}$, and arbitrary initial voltages $V_{C_{b}}(0)=11 \mathrm{~V}, V_{C_{n}}(0)=11 \mathrm{~V}$, and $V_{C_{p}}(0)=11 \mathrm{~V}$. The observer algorithms detailed in (11) and (12) are then used by the dSPACE system to monitor the effect that the current demand of Fig. 5 has on a fully charged well-conditioned battery.

Again, after $100 \mathrm{~s}$, and at regular subsequent intervals (10 s in this case), the parameter estimation algorithm is used to supply new battery model parameters.

The resulting observer estimates of $V_{C_{n}}$ are postprocessed using (2) to provide a direct comparison to $V_{C_{b}}$ (indicating the variation of SoC), which is then filtered with a low-pass filter (cutoff frequency $\omega_{c}=0.00628 \mathrm{rad}^{-1}$ ). The results of this process are shown in Fig. 15 and clearly demonstrate the ability of the observer to correct for erroneous initial conditions. Specifically, Fig. 15(a) and (b) shows SoC voltages commensurate with those expected from a fully charged battery (i.e., $\sim 13.3 \mathrm{~V}$ terminal voltage) discharging at an almost constant rate until deep discharge is encountered, and the battery health declines more rapidly.

For comparison, the cell-discharge characteristic of Fig. 11 is used to provide an estimate of the SoC voltage using more traditional current integration methods, where it can be seen that initial voltage conversion of the mapped model shows improvements over that of Randles' model, and greater Randles' model instability can only be seen [also evident in the estimated parameters of Figs. 12(a) and 14(a)] as the battery enters the deep-discharge zone. However, this may be attributed to the simple sliding-mode correction action of the Utkin observer.

In the next section, the more complicated KE is investigated, whose correction mechanism is itself adaptive in nature, allowing the discrepancies between modeling techniques to be more readily identified.

\section{Kalman Estimator TheORY}

\section{A. Underlying Theory}

The KE [11] is considered particularly useful for this application since it optimally estimates states affected by broadband noise within the system bandwidth. More generally, the use of recursive predictor/corrector type algorithms is also of benefit here, since it allows for updated parameter estimates to be readily inserted to "assist" the KE to converge to the correct voltage.

The realization of the KE follows the treatment given in [7], whereby a discrete-time equivalent model (as used in the subspace parameter estimation) of a system's state-variable description (13) is generated using a first-order Taylor series expansion, resulting in (14). Thus

$$
\begin{aligned}
\dot{x}(t) & =A x(t)+B u(t), \quad y(t)=C x(t) \\
x_{k+1} & =A_{d} x_{k}+B_{d} u_{k}, \quad y_{k+1}=C_{d} x_{k+1}
\end{aligned}
$$




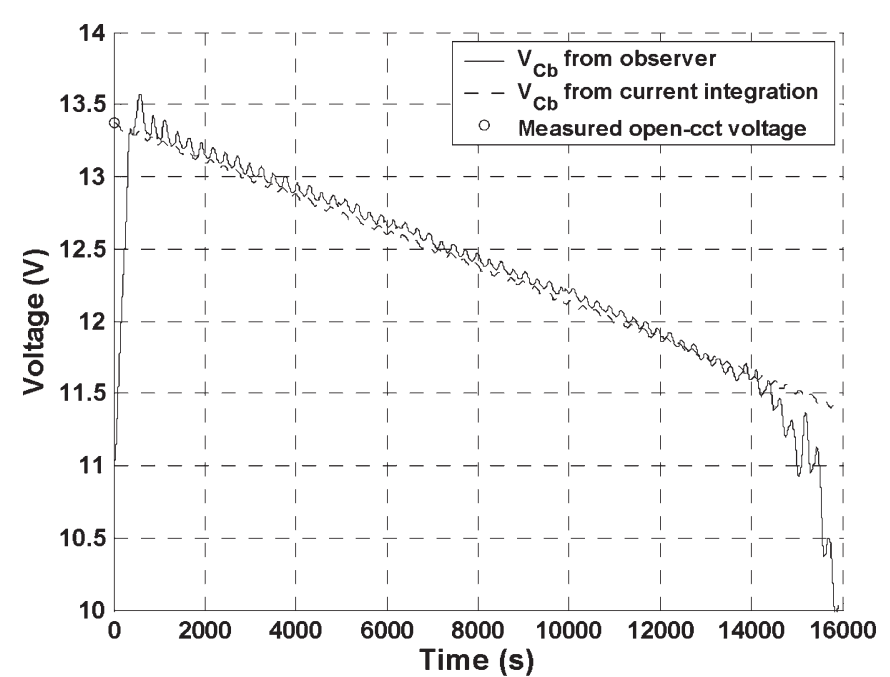

(a)

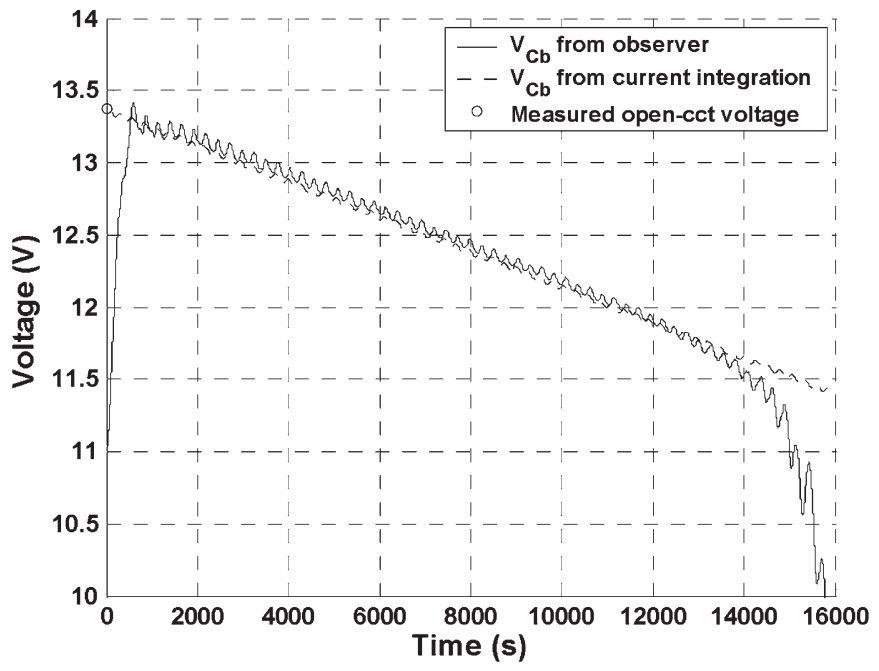

(b)

Fig. 15. Estimated $V_{C_{b}}$ showing convergence of the Utkin observer to the correct initial condition for (a) Randles' and (b) mapped models.

where

$$
A_{d} \approx I+A T_{c} \quad B_{d} \approx B T_{c} \quad C_{d} \approx C
$$

using the sampling period $T_{c}$.

This system is assumed to be corrupted by stationary Gaussian white noise consisting of a system disturbance additive vector $\sigma_{k}$ and a model disturbance additive vector $\mu_{k}$, both of which are considered to have a zero mean value for all $k$ and have the following respective covariance matrices:

$$
\mathrm{E}\left[\sigma_{k} \sigma_{k}^{T}\right]=Q \quad \mathrm{E}\left[\mu_{k} \mu_{k}^{T}\right]=R \quad(\text { for all } k)
$$

where $\mathrm{E}$ is the expectation operator. This allows the physical system to be modeled by

$$
x_{k+1}=A_{d} x_{k}+B_{d} u_{k}+\Gamma \sigma_{k}, \quad z_{k+1}=C_{d} x_{k+1}+\mu_{k+1}
$$

where $z$ is used to describe the measured outputs (e.g., $V_{o}$ incorporating the Gaussian noise), and $\Gamma$ represents the coupling between the model disturbances on each state.

The KE is implemented using the state-space equations of (3) and (4), the prediction/correction algorithms detailed in Fig. 16, and initial conditions and the covariance matrices of (18). The sample time is chosen to be $T_{c}=1 \mathrm{~s}$. Thus

$$
\begin{aligned}
P_{0} & =\left[\begin{array}{cc}
10 & 0 \\
0 & 10
\end{array}\right], & Q & =\left[\begin{array}{cc}
0.01 & 0 \\
0 & 0.1
\end{array}\right] \\
R & =10, & x_{0 / 0} & =\left[\begin{array}{l}
13.3 \\
13.3
\end{array}\right] .
\end{aligned}
$$

\section{B. Results for KE SoC Estimation Using Randles' and Remapped Models}

Results from the KE are initially preprocessed to remove the effects of the voltage dropped across $R_{i}$ (known from parameter estimation), allowing one of the states for the mapped circuit model $\left(V_{C_{p}}\right)$ to be immediately calculated, giving greater prediction accuracy over the Randles' model.

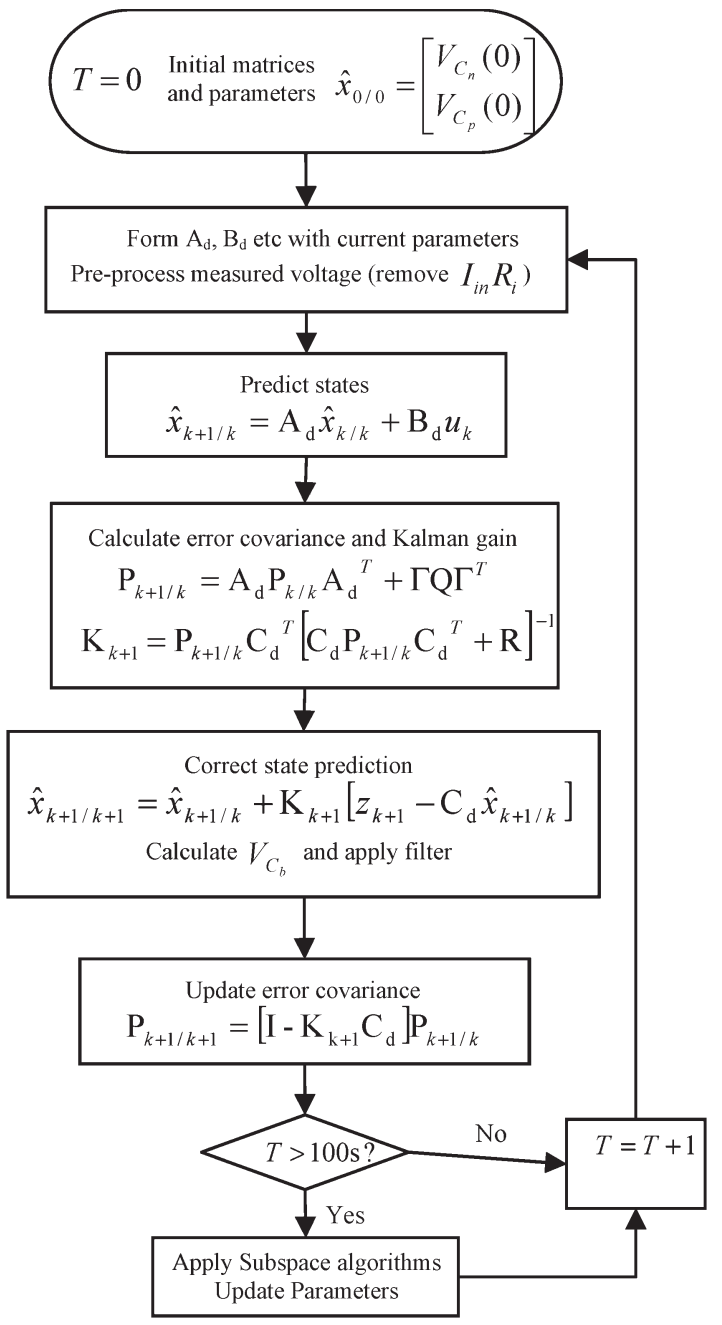

Fig. 16. Recursive implementation of the KE.

The KE is initialized with $R_{i}=6 \mathrm{~m} \Omega, R_{t}=10 \mathrm{~m} \Omega, R_{d}=$ $5 \mathrm{k} \Omega, C_{s}=2000 \mathrm{~F}, C_{b}=100000 \mathrm{~F}, V_{C_{s}}(0)=0 \mathrm{~V}, R_{n}=$ $10 \mathrm{~m} \Omega, R_{p}=5 \mathrm{k} \Omega, C_{p}=2000 \mathrm{~F}, C_{n}=100000 \mathrm{~F}$, and arbitrary initial voltages $V_{C_{b}}(0)=11 \mathrm{~V}, V_{C_{n}}(0)=11 \mathrm{~V}$, and 


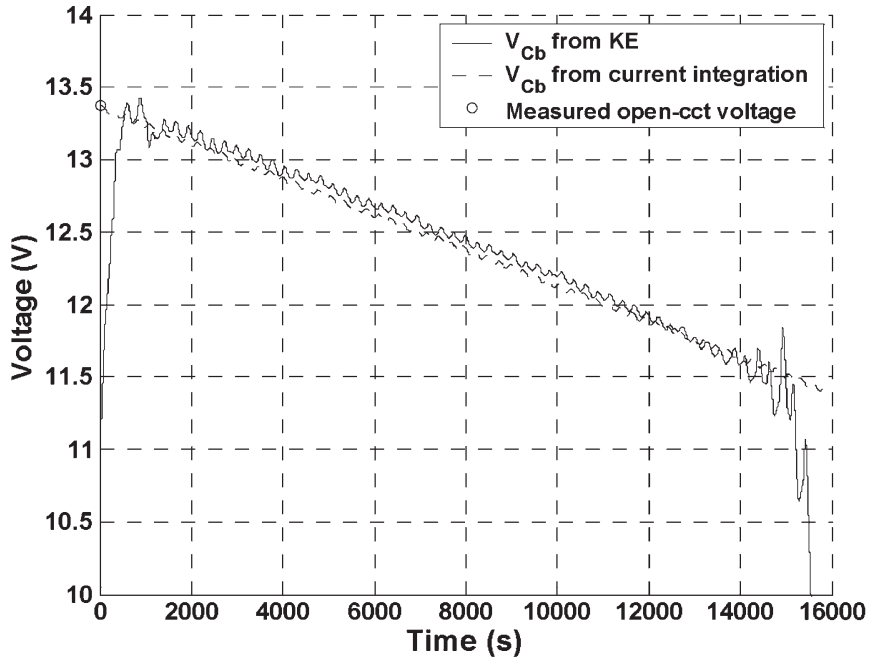

(a)

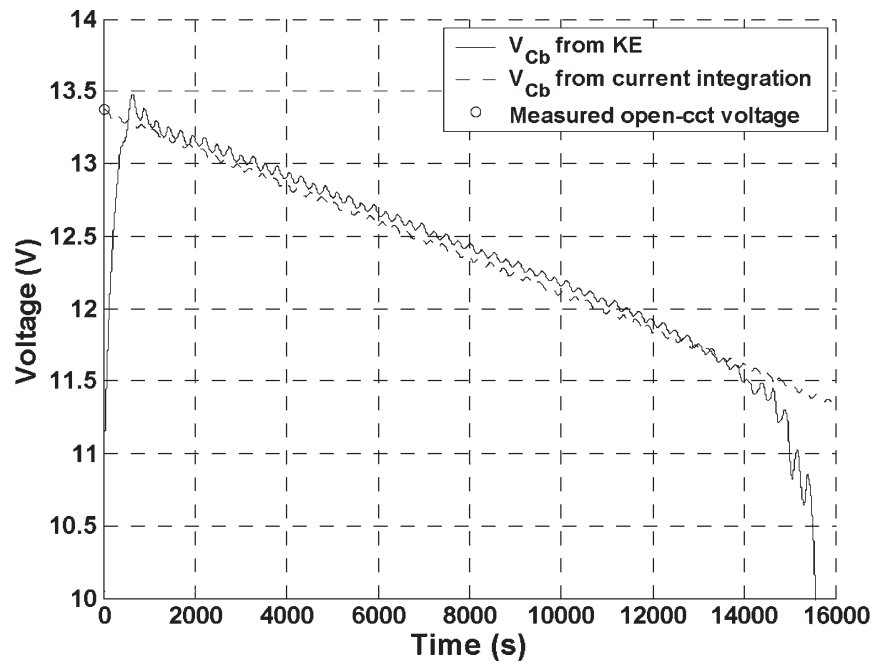

(b)

Fig. 17. Estimated $V_{C_{b}}$ showing convergence of the KE to the correct initial condition using (a) Randles' and (b) mapped models.

$V_{C_{p}}(0)=11 \mathrm{~V}$ and again used to monitor the effect that the current demand (see Fig. 5) has on a fully charged wellconditioned battery with the parameters being updated online every $10 \mathrm{~s}$.

The KE results for $V_{C_{n}}$ and $V_{C_{p}}$ are again converted using (2) to be compared with $V_{C_{b}}$ after being passed through the same low-pass filter $\left(\omega_{c}=0.00628 \mathrm{rad}^{-1}\right)$ as before. The results are shown in Fig. 17, where it can again be seen that the KE output converges to the correct SoC voltages (in this case compared with a current integration technique, which required a priori knowledge of $C_{b}$ and $\left.V_{C_{b}}(0)\right)$.

It has therefore been shown that both models exhibit excellent initial convergence; yet, it is evident that the Randles' model shows incorrect convergence due to erroneous parameter estimation for operation at low SoC (i.e., after 12000 s). Conversely, the advantageous feature of the remapped equivalent circuits readily identifiable parallel state $\left(V_{C_{b}}(0)\right)$ has allowed improved parameter estimation accuracy and, consequently, better voltage-convergence performance.

\section{State-of-Health Monitoring}

To monitor the SoH of the battery, it will be shown that $C_{b}$ may be estimated by applying a linear curve fit to buffered estimates of $V_{C_{b}}$ and considering the integrated current through $C_{b}$ over the buffer periods (typically $1800 \mathrm{~s}$ to provide stable results). Due to the convergence benefits and parameter stability afforded by the mapped circuit model (explored in Section V), it can be used to estimate the variation of $C_{b}$ (see Fig. 18) in the data presented in Fig. 17(b) - note that the data are filtered with a low-pass filter (designed with a cutoff frequency $\omega_{c}=0.0044 \mathrm{rad}^{-1}$ ).

It can be seen from Fig. 18 that, after the initial convergence of the parameter estimation and $\mathrm{KE}$ algorithms, the value of $C_{b}$ settles to an almost constant average value over the period 3000-10000 s (i.e., the typical region of operation between $\sim 80 \%$ and $\sim 30 \%$ SoC), facilitating the real-time monitoring of the $\mathrm{SoH}$ indicator. A relationship between the indicator and the

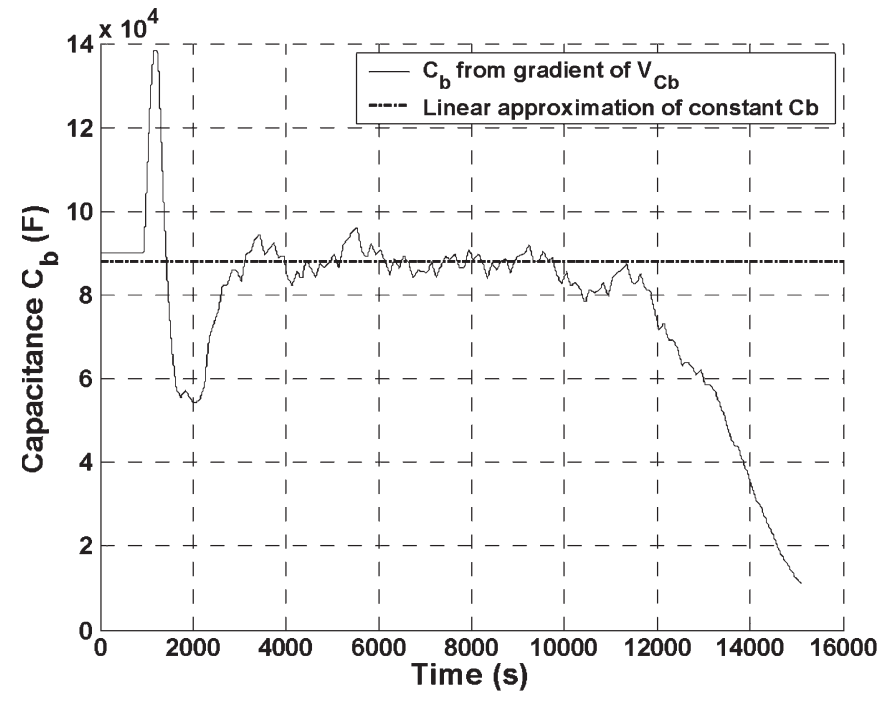

Fig. 18. Variation of $C_{b}$ from gradient of observed $V_{C_{b}}$ of Fig. 17(b).

available capacity can be obtained by making linear approximations over typical operating regions during accelerated aging tests, whereby the battery is charged with a constant $10 \mathrm{~A}$ and then discharged by the current profile shown in Fig. 5.

The resulting degradation of the $\mathrm{SoH}$ indicator $\left(C_{b}\right)$ can then be seen over time [for instance, see Fig. 19(a)], and a linear relationship is applied. Similarly, as each test is conducted, the total current demand can be integrated, allowing an estimate of the total available capacity of the battery during each test. The reduction in the available capacity of the battery under test is shown in Fig. 19(b). These relationships are approximately modeled by the following linear expressions, where $N$ is the test number:

$$
\begin{aligned}
& C_{b}=-248 N+93000 \\
& \mathrm{Ah}=-0.0625 N+48.8
\end{aligned}
$$

By manipulating (19), (20) relates the total available capacity of a battery under test to the estimated average value of $C_{b}$ 


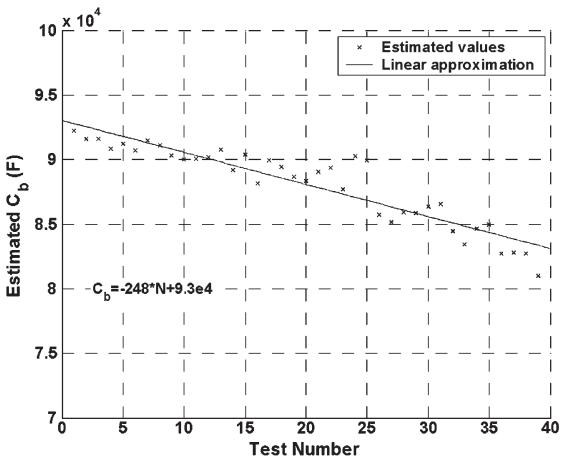

(a)

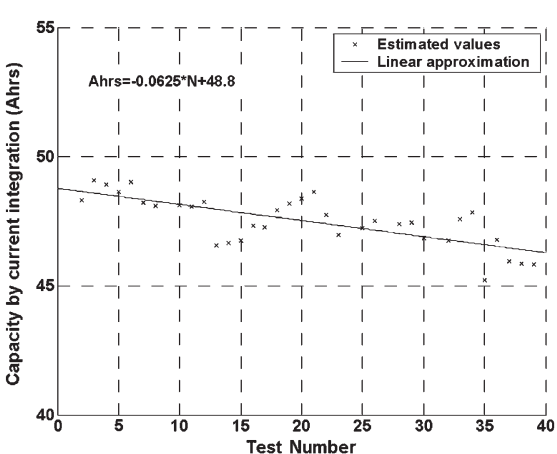

(b)

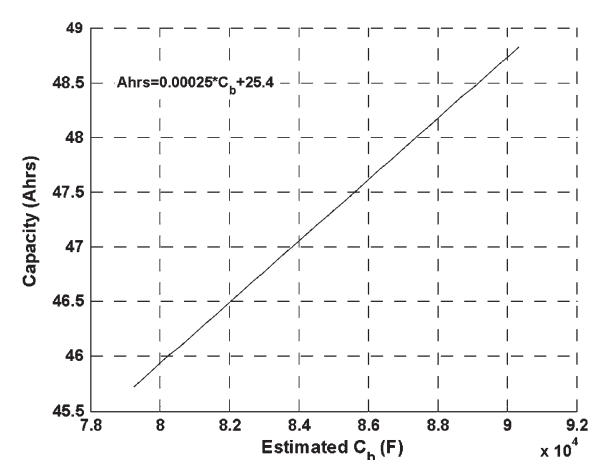

(c)

Fig. 19. (a) Decline in estimated $C_{b}$ and (b) reduction of available capacity with "age" of the battery and (c) relationship between $C_{b}$ and available capacity.

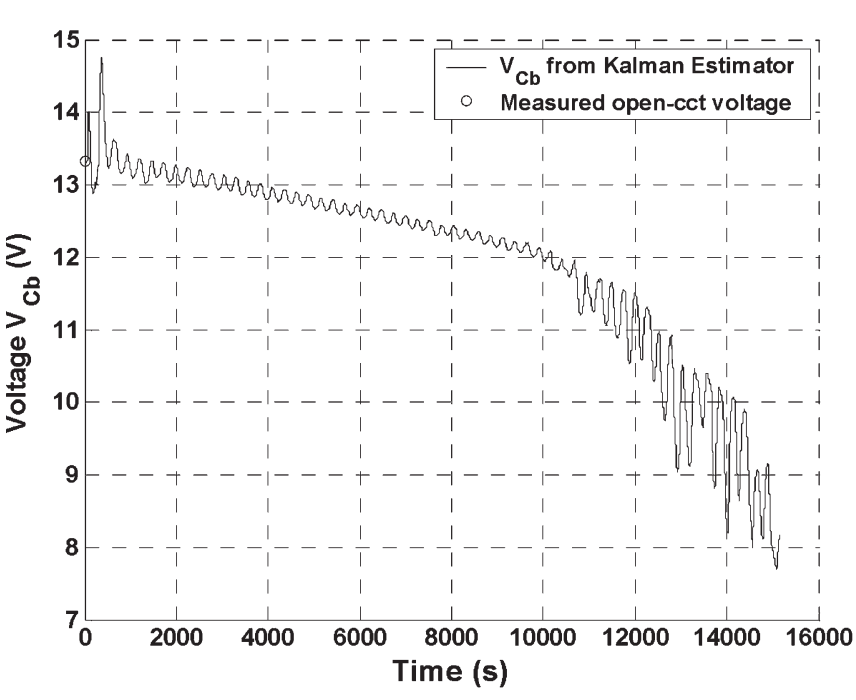

(a)

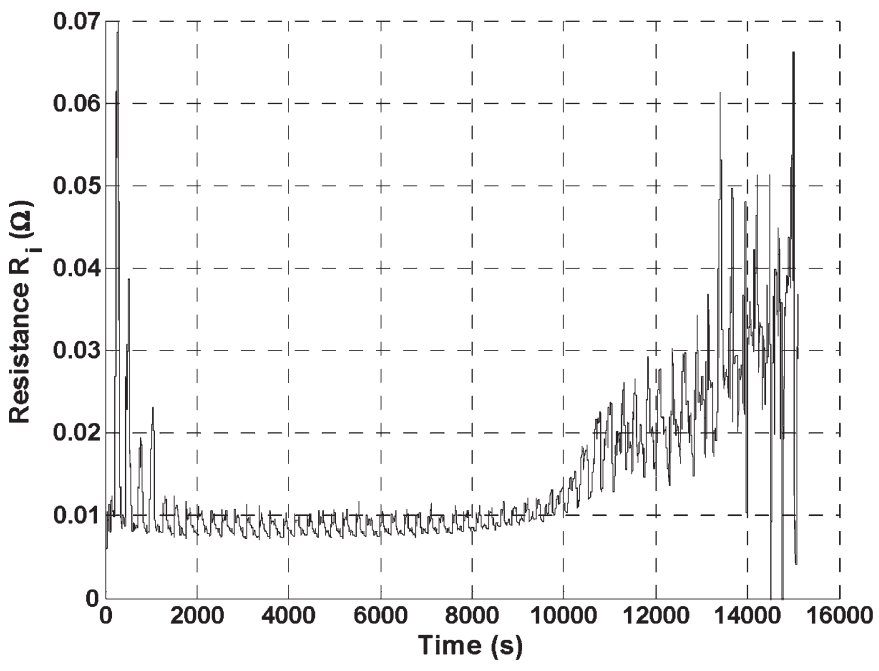

(c)

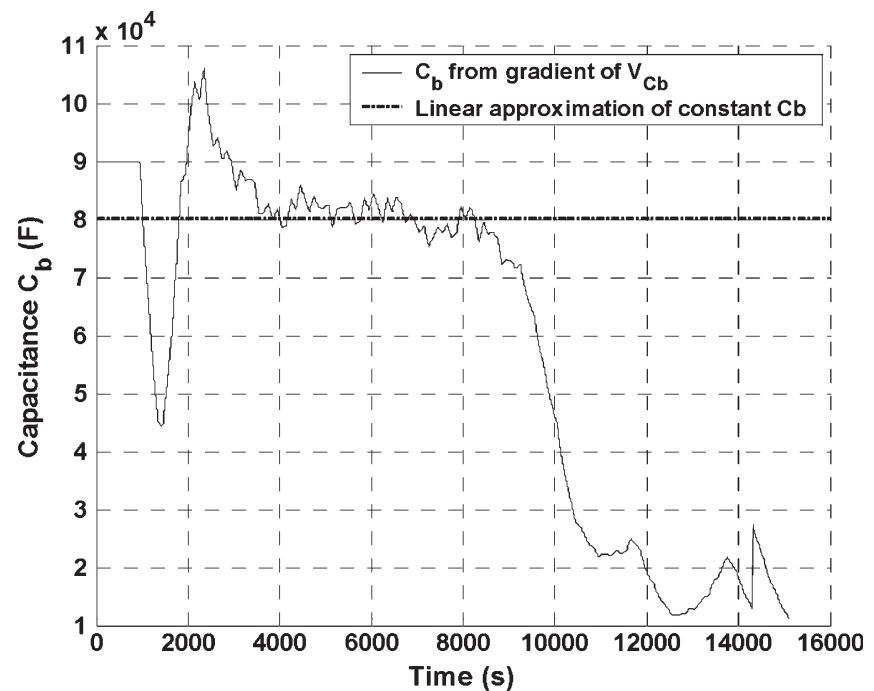

(b)

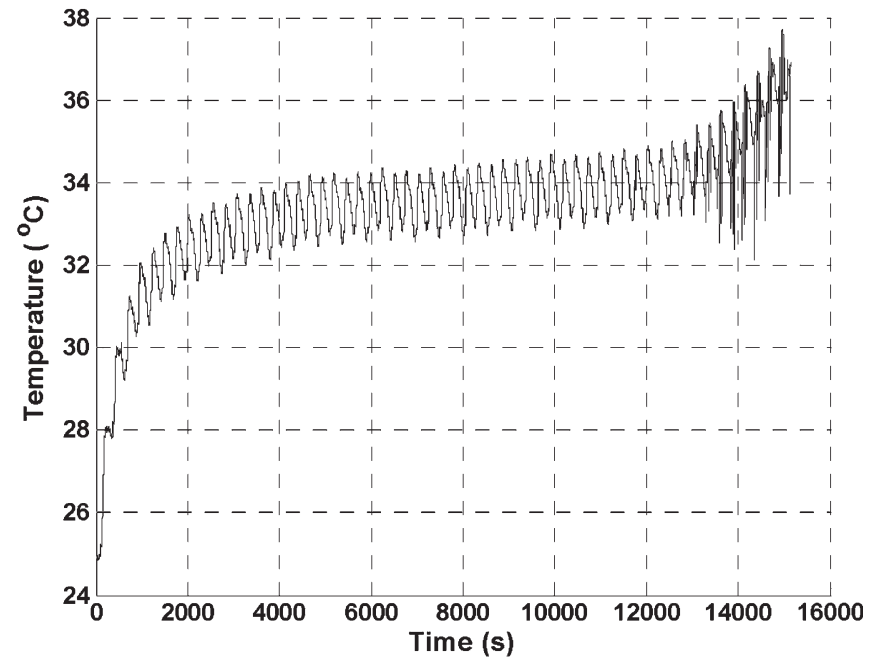

(d)

Fig. 20. (a) Estimated $V_{C_{b}}$, (b) variation of $C_{b}$, (c) variation in parameter $R_{i}$, and (d) battery terminal temperature for an "aged" battery with reduced capacity.

[see Fig. 19(c)] so that not only the relative SoH can be estimated but the total capacity of the battery at that particular time as well, i.e.,

$$
\mathrm{Ah}=0.00025 C_{b}+25.4 .
$$

This, in turn, allows more accurate current-integration $\mathrm{SoC}$ techniques to be employed (now that the necessary a priori knowledge can be estimated) and corrected using the convergence features of the KE algorithms to ensure that correct $V_{C_{b}}$ and $\mathrm{SoC}$ estimates are obtained. 
Again, by way of example, for the case of a battery with SoC and SoH shown in Figs. 17(b) and 18, respectively, the estimated $C_{b}$ of $88500 \mathrm{~F}$ from Fig. 18 maps to an available capacity of $47.5 \mathrm{Ah}$, as compared with that of $47.4 \mathrm{Ah}$ achieved by integrating the total current demand. The battery is therefore shown to be in good health.

In a similar manner to that used to generate the results of Fig. 17, Fig. 20 now shows the estimated variation of $V_{C_{b}}$ and $C_{b}$ for the same battery after months of continuous repetitive cycling. A linear approximation to the variation in $C_{b}$ indicates that the capacitance of the main charge store has degraded from 88500 F (see Fig. 18) to 80200 F [see Fig. 20(b)]. Consequently, the available capacity can be determined from (20) to have decreased from 47.5 to $45.4 \mathrm{Ah}$, whereas integration of the current demand over the length of this particular cycle results in a net removal of 45.8 Ah of charge.

This therefore shows that the SoH of this battery is deteriorating with use (since a decrease in the value of $C_{b}$ indicates a diminished plate/grid surface area [9] or a loss of electrolyte), and careful monitoring of any rapid change in the estimated parameter values [see Fig. 20(c)] is advisable to preempt a rapid increase in battery temperature [see Fig. 20(d)], which could indicate thermal runaway (leading to excessive hydrogen production), short-circuits or plate/anodic deterioration (hence increased internal resistance $R_{i}$ ), and, ultimately, catastrophic failure of the battery.

\section{CONCLUSION}

A novel battery-modeling methodology for an all-electric PRT vehicle has been proposed for the purpose of battery $\mathrm{SoH} / \mathrm{SoC}$ estimation. The methodology consists of the use of a novel battery circuit model, a predictor/corrector observer, and online subspace parameter estimation, allowing the observer to adaptively estimate and converge on voltages associated with battery functionality indicators. The adaptive nature of the algorithms allows the effects of ambient temperature variations and battery self-discharge issues to be inherently accommodated. Moreover, the resulting parameter estimates are used to identify fault conditions and areas of unsafe operation. Experimental results show improved voltage predictions compared with algorithms based on the traditional Randles' model and facilitate decisions based on SoC and $\mathrm{SoH}$ to be made. A relationship between a battery's available capacity and the estimated capacitance of the main charge store has also been found and shows excellent agreement with experimental results.

\section{ACKNOWLEDGMENT}

The authors would like to thank Advanced Transport Systems Ltd. for their cooperation, particularly N. Zurlinden, for providing the ULTRA driving cycle data and press shots.

\section{REFERENCES}

[1] [Online]. Avalable: http://www.atsltd.co.uk

[2] A. Jossen, "Fundamentals of battery dynamics," J. Power Sources, vol. 154, no. 2, pp. 530-538, Mar. 2006.
[3] A. Kawamura and T. Yanagihara, "State of charge estimation of sealed lead-acid batteries used for electric vehicles," in Proc. 29th Annu. IEEE Power Electron. Spec. Conf., May 1998, vol. 1, pp. 583-587.

[4] P. Sinclair, R. Duke, and S. Round, "An adaptive battery monitoring system for an electric vehicle," in Proc. Int. Conf. Power Electron. Drives Energy Syst. Ind. Growth, Dec. 1998, vol. 2, pp. 786-791.

[5] P.-S. Kim, "New estimation filtering for battery management systems of lead-acid cells in hybrid electric vehicles," Int. J. Comput. Sci. Netw. Security, vol. 7, no. 2, pp. 136-141, Feb. 2007.

[6] C. S. C. Bose and F. C. Laman, "Battery state of health estimation through coup de fouet," in Proc. 22nd INTELEC, 2000, pp. 597-601.

[7] B. S. Bhangu, P. Bentley, D. A. Stone, and C. M. Bingham, "Observer techniques for estimating the state-of-charge and state-of-health of VRLABs for hybrid-electric vehicles," in Proc. IEEE Conf. Veh. Power Propuls., Sep. 2005, pp. 780-789.

[8] D. Berndt, E. Meissner, and R. Rusch, "Aging effects in valveregulated lead-acid batteries," in Proc. 15th INTELEC, Sep. 1993, vol. 2, pp. 139-145.

[9] P. Ruetschi, "Aging mechanisms and service life of lead-acid batteries," J. Power Sources, vol. 127, no. 1/2, pp. 33-44, Mar. 2004.

[10] K. J. Vetter, Elecktrochemische Kinetik. Berlin, Germany: SpringerVerlag, 1961, p. 268.

[11] R. E. Kalman, "A new approach to linear filtering and prediction problems," Trans. ASME—J. Basic Eng., Ser. D, vol. 82, no. 1, pp. 35-45, 1982.

[12] L. Ljung, System Identification-Theory for the User, 2nd ed. Englewood Cliffs, NJ: Prentice-Hall, 1999, pp. 317-353.

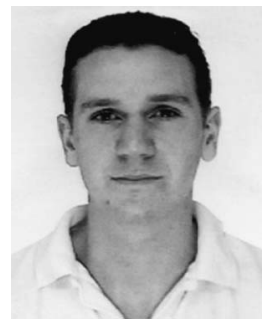

C. R. Gould received the M.Eng. degree in electronic and electrical engineering with communications and the Ph.D. degree from the University of Sheffield, Sheffield, U.K, in 2002 and 2006, respectively.

Since 2005, he has been a Research Associate with the Electrical Machines and Drives Group, Department of Electronic and Electrical Engineering, University of Sheffield. His current research interests include the modeling and control of resonant power supplies, battery-management systems and battery state-of-charge/health modeling for all-electric steer-by wire vehicles, and the design of efficient brushless ac motor for sensorless control of electric mobility vehicles.

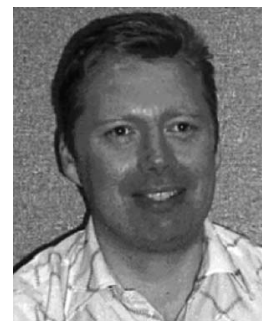

C. M. Bingham (M'00) received the B.Eng. degree in electronic systems and control engineering from Sheffield City Polytechnic, Sheffield, U.K., in 1989, the M.Sc. (Eng.) degree in control systems engineering from the University of Sheffield in 1990, and the Ph.D. degree from Cranfield University, Shrivenham, U.K., in 1994, for research on control systems to accommodate nonlinear dynamic effects in aerospace flight-surface actuators.

He was a Postdoctoral Researcher with Cranfield University until taking a research position with the University of Sheffield. He was appointed Lecturer with the Department of Electronic and Electrical Engineering, University of Sheffield, in 1998 and Senior Lecturer in 2003. His current research interests include traction control/ antilock braking systems for electric vehicles, electromechanical actuation of flight control surfaces, control of active magnetic bearings for high-speed machines, sensorless control of brushless machines, and the high-speed modeling, analysis, and design of resonant power converters. 


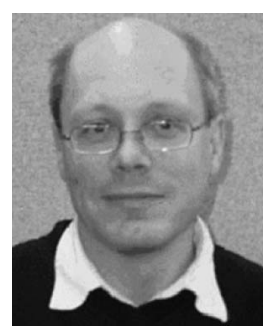

D. A. Stone received the B.Eng. degree in electronic engineering from the University of Sheffield, Sheffield, U.K., in 1984 and the Ph.D. degree from Liverpool University, Liverpool, U.K., in 1989.

$\mathrm{He}$ is currently with the University of Sheffield as a Member of Academic Staff, specializing in power electronics and machine drive systems. His current research interests include hybrid-electric vehicles, battery charging, electromagnetic compatibility, and novel lamp ballast for low-pressure fluorescent lamps.

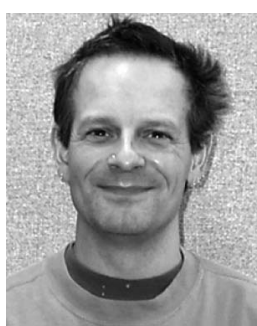

P. Bentley received the B.Sc. degree in engineering physics from Sheffield Hallam University, Sheffield, U.K., in 1998 and the Ph.D. degree for the dynamic characterization and modeling of battery technologies for all/more electric vehicles from the University of Sheffield in 2004.

He has been a Research Associate with the Electrical Machines and Drives Group, Department of Electronic and Electrical Engineering, University of Sheffield, since 2001. His research interests include energy storage components for hybrid and electric vehicle drive trains and energy-scavenging devices. 